\title{
POTENTIAL OF PHASE MEASUREMENTS IN RADAR INTERFEROMETRY FOR THE OBSERVATION OF EMERGENCY SITUATIONS BUREYA LANDSLIDE CASE
}

\author{
Alexander I. Zakharov, Liudmila N. Zakharova \\ Kotelnikov Institute of Radioengineering and Electronics of RAS, Fryazino branch, http://fire.relarn.ru \\ Fryazino 141190, Moscow Region, Russian Federation \\ aizakhar@ire.rssi.ru,lu@ire.rssi.ru
}

Abstract. Results of the European spaceborne Sentinel-1 synthetic aperture radar differential interferometry data processing for the Bureya landslide area aimed at detection and assessment the consequences of catastrophic event in December 2018 are presented. More than 20 scenes, obtained in SAR repeated orbits observations from relative orbits 134 and 61 for the period from December 2018 to March 2019 having high temporal stability of signal backscatter from landslide slope, were used. Analysis of phase measurements on interferograms with small spatial baselines and on differential interferograms (in the case of large interferometric baseline) allowed us to make conclusion about temporal stability of the landslide circus surface during the entire period covered by the Sentinel-1 SAR observations being analyzed. No noticeable (more than few millimeters) small-scale displacements of the slopes in the end of January 2019 due to blasting operations in the riverbed cofferdam were revealed. We estimated landslide scales also. The volume of the ground that slid down the riverbank slope was at least 18.5 million $\mathrm{m} 3$, maximal vertical displacement of the scattering surface after the landfall compared with SRTM digital elevation model reached 140-150 $\mathrm{m}$, the landslide area in the vertical projection is $22.8 \mathrm{ha}$. Thus, Sentinel-1 radar data demonstrated its potential to monitor the stability of landslide slopes by means of radar interferometry technique and high-accuracy of the measurements of probable small-scale surface displacements provided the conduction of the radar observations in the winter time period.

Keywords: landslide, synthetic aperture radar (SAR), SAR interferometry, surface displacement measurement, digital elevation model

UDC 621.396.96

Bibliography - 16 references

Received 19.03.2019, accepted 17.04.2019

RENSIT, 2019, 11(1):31-38

DOI: 10.17725/rensit.2019.11.031

Contents

1. INTRODUCTION (31)

2. Technique fundamentals (32)

3. Data used in analysis (34)

4. Processing and Discussion Results (34)

5. Conclusion (37)

References (37)

\section{INTRODUCTION}

Catastrophic landslide on the Bureya riverbank in December 2018 dammed the riverbed and put nearby settlements and residential/ industrial infrastructure objects located downstream the dam at risk of flooding and destruction because of foreseeable break of the natural dam in spring. According to multispectral sensor of Japanese geostationary satellite Himawari-8, the landfall took place on December 11, 2018. First ideas about the scale of this catastrophic natural event were got from analysis of Sentinel-2 optical image made on December 12, 2018, where the landscape alterations as well as the landslide location and its spatial size are clearly seen. Field observations in January 2019 provided information about the landslide scale: its length is 800 meters long, and volume of the rock soils slipped down is about $24 \mathrm{M}$ cubic meters. To restore the water flow through the layer of soil rocks a series of explosions was carried out by military specialists; the channel formed by explosions sustained to the 
decrease of water level above the dam. Such an all-weather radar observation techniques including radar interferometry increase our understanding of scale and dynamics of alike natural phenomena.

Radar interferometry as a tool for monitoring the landslide processes is widely used since the end of last century thanks to availability of spaceborne synthetic aperture radar data (SAR) operating in X, C, L bands [3]. Numerous examples of the application of this technique demonstrate possibility of detection and estimation of amplitude of small-scale displacements of the scattering surface over the landslide body with accuracy of several millimeters, estimation the landslide square, and long-term history of the landslide process evolution [4-7]. The loss of coherence of scattered signals due to varying scattering conditions in comparatively short-wavelength SAR bands, especially when imaging surfaces covered with dense vegetation, stimulated the improvement of classical methods of differential radar interferometry, which resulted in creation of permanent scatterers technique [8-11] and its alternatives: STAMPS [12], SQUEESAR [13], small baselines technique (Small BASeline, SBAS) [14], and others. Essential requirement of permanent scatterers technique and its analogs is a requirement for joint analysis of long series of SAR observations with subsequent application of statistical analysis techniques in order to identify reliable stable scatterers. In this study, due to limited set of available radar images of the landslide territory, only classical differential radar interferometry technique will be used.

\section{TECHNIQUE FUNDAMENTALS}

Radar interferometry technique exploits an information about phase difference of the echo signals scattered from some terrain area and recorded by radar system operating at two spatially close points provided the condition of mutual coherence of the echo signals is satisfied. In this case phase difference between recorded echo signals depends on the difference of the distances from observation points to the target and contains information about the surface topography variations and about small-scale alterations of the distance till the target during time between surveys in the repeated observations scheme $[15,16]$.

An illustration of the topography observations geometry is shown in Fig. 1. The topography details, satellite orbit altitudes, and their spacing are not to scale for the clarity, the geoid reference surface in the imaging area is approximated by plane. Point $A_{1}$ is the first observation point, $A_{2}$ is second observation point; second point may be, for example, on the other trajectory of the same satellite happened some time later. Let $H$ be height of the satellite above the surface at first observation point. The distance between observation points di is called as interferometric baseline, which is oriented at an angle $\alpha$ i with respect to horizon. Radar signal transmitted from point $A_{1}$ arrives to the point $P$ on surface located at a distance $r 1$ at an angle $\alpha$ with respect to nadir direction and local vertical.

Interferogram is a map of the signals phase difference $\Delta \varphi=\varphi_{1}-\varphi_{2}$ calculated as a result of the pixel-wise complex multiplication of signals $U_{1}$ and $U_{2}$, scattered from the same surface element and acquired at the points $A_{1}$ and $A_{2}$ :

$$
U_{1} U_{2}^{*}=u_{1} u_{2} \exp \left(j\left(\varphi_{1}-\varphi_{2}\right)\right)=u_{1} u_{2} \exp \left(\frac{-j 4 \pi \Delta r}{\lambda}\right)
$$

where $u_{1}$ and $u_{2}$ are signal amplitudes, $\Delta r$ is the difference of distances from observation points to the selected surface element, $\lambda$ is wavelength.

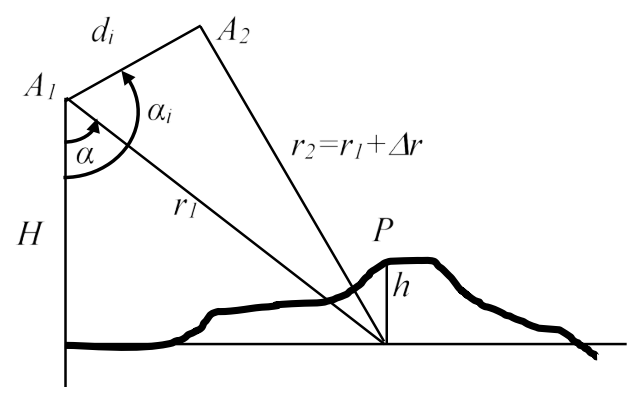

Fig. 1. Geometry of the interferometric observation of the surface topography. 
Phase difference $\Delta \varphi$ on the interferogram is a result of joint processing of a pair of radar images. It contains information about the topography (so-called topographic phase $\left.\Delta \varphi_{t}\right)$, small-scale dynamics (displacements) of the scattering surface $\Delta \varphi_{d}$ phase fluctuations along the signal propagation path in atmosphere $\Delta \varphi_{a}$, thermal noise of the SAR instrument and processing noise $\Delta \varphi_{N}$, etc. Taking into account two first components only, we can write down $[15,16]$

$$
\Delta \varphi_{12}=\Delta \varphi_{t}+\Delta \varphi_{d}=-\frac{4 \pi d_{p} \Delta h}{\lambda r \sin \alpha}-\frac{4 \pi \delta r_{d}}{\lambda},
$$

where perpendicular component of the interferometric baseline is equal to $d_{p}=d_{i} \sin \left(a_{i}-a\right)$.

Note that the sensitivity of interferometric phase difference to the heights variations $\Delta b$ increases with rise of baseline component $d_{p}$, and also with decrease of signal wavelength $\lambda$, incidence angle $a$, and slant range $r$ to the surface. For the Sentinel-1 radar, the wavelength $\lambda=5.6 \mathrm{~cm}$, slant range from radar to the surface of the landslide is $820 \mathrm{~km}$. Small-scale displacements of scattering surface during time interval between surveys is not shown in the figure due to its small size compared with the scale of the observation geometry. In the case of displacement amplitude of an order of, for example, $\delta r_{d}=0.5 \lambda$ in (1), phase difference on the interferogram will change by $2 \pi$. An important numerical indicator of the sensitivity of $\Delta \varphi_{t}$ to the topography is height ambiguity $b_{a}$ - topographic height variation between two surface points, which introduces $2 \pi$ phase shift on the interferogram:

$h_{a}=\lambda r\left(2 d_{p}\right)^{-1} \sin a$.

In order to interpret phase information on the interferogram correctly it is necessary to take into account the fact that in the case of positive $d_{p}$, according to (1), phase difference on the interferogram decreases with increase of surface height with respect to digital elevation modelused in interferometric processing chain. In the case of negative $d_{p}$ it increases. The manifestation of small-scale radial displacements of the surface during time interval between observations is such that according to (1), surface subsidence will cause positive phase difference rate on the interferogram. It is necessary to highlight that there is no possibility to measure simultaneously topography variations and small-scale radial displacements of the surface between observations. The problem of extracting dynamics of the scattering surface can be solved using differential interferometry approach, which supposes elimination of topographic phase by utilizing a reference digital elevation model (DEM), or by selection of image pairs obtained in observation geometry with very small baseline $d_{b}$, when the first (topographic) component on the right side of eq. (1) vanishes and thus may be ignored. In this study we use differential interferometry technique, and most of topographic effects were synthesized using SRTM DEM data and removed from interferograms.

Relative accuracy of topographic measurements within the image frame is determined by coherence of signals acquired at observation points $A_{1}$ and $A_{2}$ in Fig. 1. The degree of coherence of the echo signals within complex radar images $U_{1}$ and $U_{2}$ can be measured directly using radar data, it is determined by following expression:

$$
\gamma=\frac{E\left\{U_{1} \cdot U_{2}^{*}\right\}}{\sqrt{E\left\{\left|U_{1}\right|^{2}\right\} E\left\{\left|U_{2}\right|^{2}\right\}}},
$$

where $E\{\bullet\}$ is an expectation over ensemble of samples within spatial window centered at current image element.

Among the main factors affecting the level of $\gamma$ there are decorrelation $\gamma_{N}$ caused by thermal noise of radar system, spatial decorrelation $\gamma_{s p}$ of scattered signals caused by alterations of acquisition conditions of backscatter at different spatial points, temporal decorrelation of the conditions of signals backscatter $\gamma_{t d}$ due to displacement of observations in time, and, finally, volume decorrelation of signals in layered 
covers characterized by $\gamma_{i}$. Let us restrict our consideration to the effects mentioned above and assume their independence, so we may write $\gamma=\gamma_{N} \gamma_{s p} \gamma_{t d} \gamma_{v}$

IInterferometric coherence characterizes errors of the phase difference measurements and, accordingly, relative interpixel accuracy of the topographic heights measurements and displacements of the scattering surface estimation $[15,16]$.

\section{DATA USED IN ANALYSIS}

We used the data of European synthetic aperture radar Sentinel-1, acquired in IW (Interferometric Wide) mode. The width of standard frame, consisting of 3 subswaths in this mode, is 240 $\mathrm{km}$. The landslide zone lays within the Sentinel-1 frame when imaging is made from relative orbits number 61 and 134. Incidence angle of radar signal is $32^{\circ}$ in observations from relative orbit 61 (subswath No.1 of the SCANSAR Sentinel- 1 mode), it is $42^{\circ}$ for observations from relative orbit 134 (subswath No.3). Sentinel-1 orbit repetition period is 12 days, so from the moment of the landfall in early December 2018 till mid-March 2019, at least 10 imaging sessions were conducted from each of the orbits, resulting in accumulation of comparatively large datasets applicable for interferometric processing. Tables 1 and 2 contain description of observation dates for pairs of observation sessions, size of the interferometric baseline perpendicular component and height ambiguity values. Pairs of images with 12 days interval were used as primary image combinations.

Table 1.

Parameters of Sentinel-1 SAR interferometric observations from relative orbit 134 .

\begin{tabular}{|c|c|c|c|}
\hline Pair No & Observation date, yyyymmdd & $d_{p,}, m$ & $h_{a^{\prime}}, \mathrm{m}$ \\
\hline 1 & $20181128-20181210$ & 61 & 251 \\
\hline 2 & $20181210-20181222$ & 83 & 185 \\
\hline 3 & $20181222-20190103$ & -69 & 221 \\
\hline 4 & $20190103-20190115$ & -90 & 170 \\
\hline 5 & $20190115-20190127$ & 40 & 381 \\
\hline 6 & $20190127-20190208$ & 50 & 308 \\
\hline 7 & $20190208-20190220$ & 39 & 395 \\
\hline 8 & $20190220-20190304$ & -12 & 1304 \\
\hline
\end{tabular}

Table 2.

Parameters of Sentinel-1 SAR interferometric observations from relative orbit 61.

\begin{tabular}{|c|c|c|c|}
\hline Pair No & Observation date, yyyymmdd & $d_{p,}, m$ & $h_{a}, \mathrm{~m}$ \\
\hline 1 & $20181123-20181205$ & 34 & 520 \\
\hline 2 & $20181205-20181217$ & -151 & 118 \\
\hline 3 & $20181217-20181229$ & 8 & 2222 \\
\hline 4 & $20181229-20190110$ & 51 & 351 \\
\hline 5 & $20190110-20190122$ & 16 & 1096 \\
\hline 6 & $20190122-20190203$ & 81 & 220 \\
\hline 7 & $20190203-20190215$ & -149 & 120 \\
\hline 8 & $20190215-20190227$ & 9 & 1957 \\
\hline 9 & $20190227-20190311$ & -30 & 594 \\
\hline
\end{tabular}

Interferometric pairs with larger time interval between observations are typically characterized by extremely low scattered signals coherence due to temporal decorrelation $\gamma_{t d}$ in formula (3) and, as a result, by loss of informational content in phase measurements.

We used data in the SLC (Single Look Complex) format. Spacing of image pixels in slant range in this format is $2.3 \mathrm{~m}$, lines spacing is $14.1 \mathrm{~m}$. During interferometric processing the data averaging in range (4 pixels) was applied for reducing noise level. As a result of averaging, the pixels spacing in azimuth and ground range are about the same in the interferogram image.

\section{PROCESSING AND DISCUSSION RESULTS}

Interferometric coherence is an important factor that determines noise level in the interferogram, the accuracy of measurements of the topography and displacements of the underlying surface. Quality of interferometric measurements with $C$-band radars is significantly affected by temporal decorrelation of the scattered signals. The shorter time interval between observations and the lower biomass of vegetation in the survey area, the higher the coherence. There is seasonal variation of quality of measurements: in winter time the coherence tends to increasing due to larger stability of the signal scattering conditions of frozen soil and vegetation. Coherence of signal backscattered 


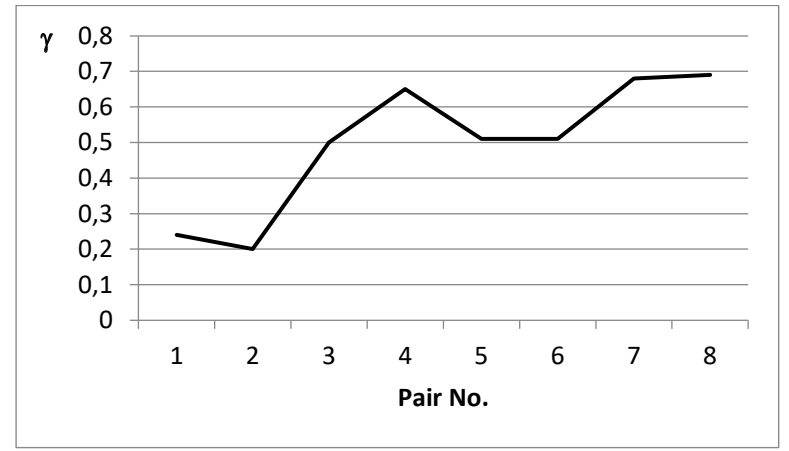

Fig. 2. Coherence of the signals scattered by the landslide for image pairs from Table 1.

from the landslide surface for interferometric pairs from the Table 1 is plotted in Fig. 2

The fact that the first sample in the plot have low coherence level can be explained by high variability of the signal scattering conditions in the case of unfrozen covers. The next, even lower, coherence value for the pair 20181210-20181222, made in frozen conditions according to meteorological data, may be explained by completely modified micro-relief of the landslide surface after the landfall took place. Since the third data pair of observations (after December 22, 2018), frozen soil and vegetation cover in the survey area become a more stable reflective media, and for that reason it is possible to evaluate new topographic shape in the landslide zone and to estimate latter smallscale soil movements, if any.

The detail on the interferogram that is characteristic of a landslide area in the case of large $d_{p}$ and, consequently, a good sensitivity of the phase measurements to the topography variations is clearly distinguishable oval structure in Fig. $\mathbf{3} b$ (interferometric pair with a positive baseline component $d_{p}$ ) and $3 c$ (interferometric pair with a negative $d_{\mathrm{p}}$ ). Current topography of the landslide circus differs from digital elevation model used to compensate topography on the interferogram. Because of the relief underestimation in this instance, the residual topographic phase may be observed on the interferogram - in the first case the phase level increases from edge of the circus to its conditional center (the brightness of the phase image increases in accordance with the adopted encoding, Fig. 3b). In the second case, since interferometric baseline has opposite sign, the phase difference decreases, the brightness decreases. Similar phase details on the interferograms are true for all the pairs of sessions from Tables 1 and 2 up to midMarch 2019, excluding pairs of sessions with small interferometric baselines, what allows us to make an assumption about domination of the topography in these interferometric pairs. It should be noted that since surface subsidence is always going in the same direction relative to the radar; it produces monotonous increase of the phase difference, according to (1). In both interferograms of Fig. 3 phase jump by $2 \pi$, clearly visible in the right side of the oval circus feature, is absent in its left side. That is due to the effect of distortion of radar images in side looking observation geometry, when surface slopes tilted towards the antenna are foreshortened. In

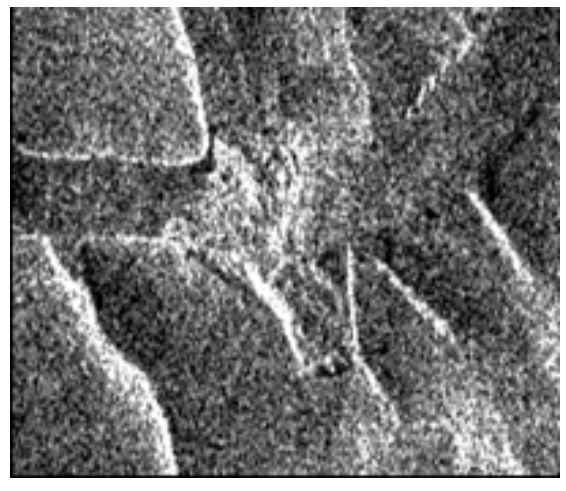

(a)

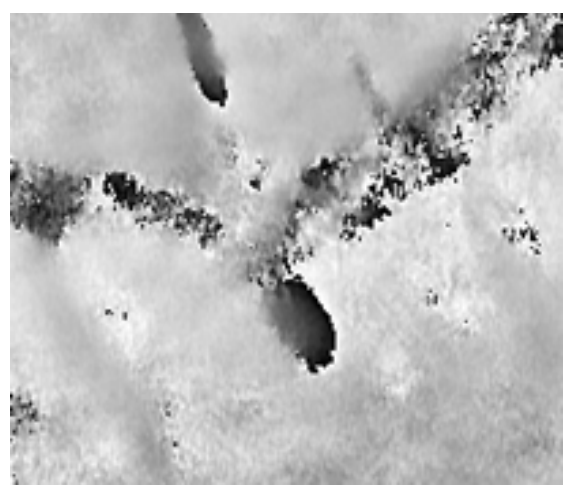

(b)

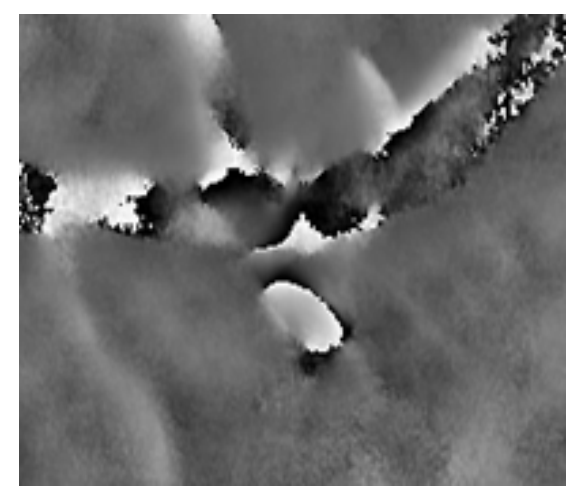

(c)

Fig. 3. Amplitude image (a), interferogram with positive $d_{p}(b)$, and interferogram with negative $d_{p}(c)$. 


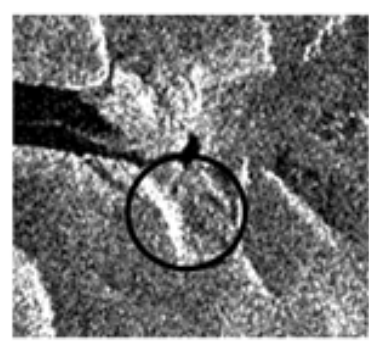

(a)

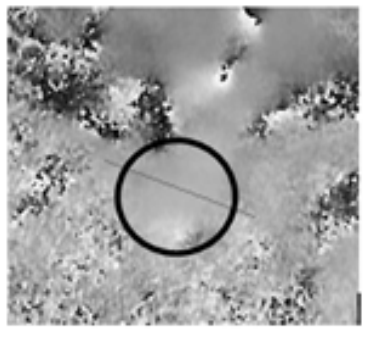

(b)
Fig. 4. Amplitude image (a) and interferogram (b) for a pair of the images with small baseline.

Fig. 3 radar looks to the left. Thus, the left edge of the landslide depression is foreshortened, and it is presented on the amplitude image as a bright narrow line (Fig. 3a). For that reason, it is impossible to track the phase rate across distal edge of circus on the interferogram. Because of pixels averaging in range during processing as well as filtering of the interferogram the final shape of circus on the image in a form of open ring may be seen in Figs. 3b and 3c.

When solving the problem of detecting the dynamics of the scattering surface between repeated observations, one of the ways to reduce the effect of topographic errors on the interferogram is a careful selection of the image pairs with proper interferometric baselines: selection of image pairs with small baselines allows to neglect the $\Delta \varphi_{t}$ component in (1). In our case, the pairs 1 and 8 from Table 1 and pairs 3, 5,8 from Table 2 meet this requirement. Because of short baseline (about $15 \mathrm{~m}$ ) and respective height ambiguity of about $1000 \mathrm{~m}$ (see, for example, a pair 5 from table 2), the topographic height error $\Delta h=100 \mathrm{~m}$ in (1) will cause to the same phase deviation on the interferogram, as a

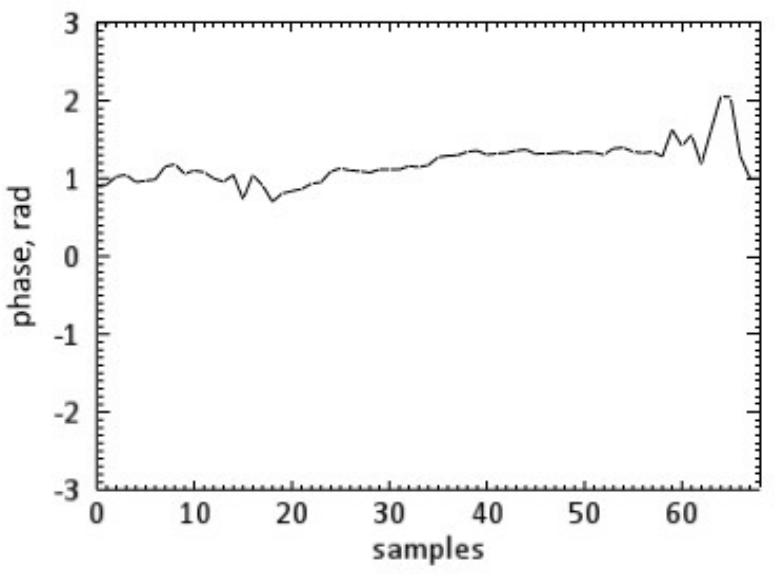

Fig. 5. Plot of phase difference values along the line in Fig. 4b. small-scale surface radial displacement by $\lambda / 20$, that is only $2.8 \mathrm{~mm}$. Typical interferogram for such an interferometric pair (a pair of sessions on February 15-27, 2019) is shown in Fig. 4. On the phase difference profile crossing the landslide in the transverse direction (Fig. 5, the position of the profile line is shown in Fig. $4 \mathrm{~b}$ ), there are no noticeable phase variations, except for minor fluctuations of an order of 0.5 radians. Possible displacement of the surface corresponding to this phase variation would not exceed $2 \mathrm{~mm}$. Similar results were obtained with pairs of images on December 17-29, 2018 (8 m baseline) and December 10-22, 2019 (16 $\mathrm{m}$ baseline), whence it follows that there was no noticeable small-scale surface dynamics in December, in mid-January and late February

Time interval since January 22 till February 1, 2019 with active blasting operations is covered with image pairs having relatively large interferometric baselines, and therefore in order to compensate the presence of the circus topographic phase, the phase on the

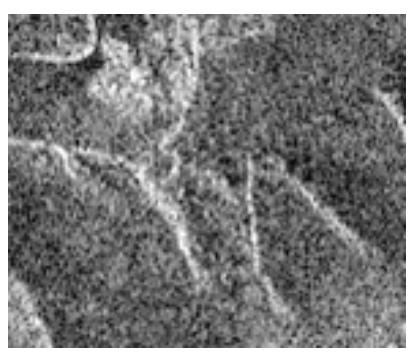

(a)

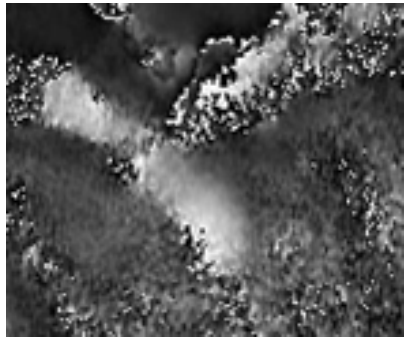

(b)

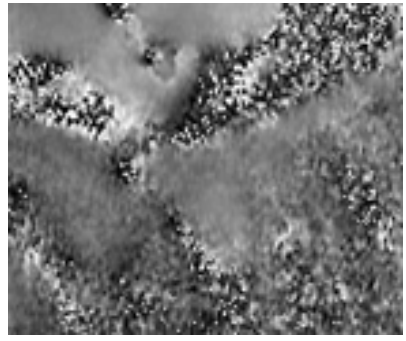

(c)

Fig. 6. Amplitude image (a), interferogram before correction of topographic phase (b) andcorrected interferogram. 
interferograms of pairs 5 and 6 from Table 1 , as well as pair 6 from Table 2, was corrected for the phase component $\Delta \varphi$, caused by circus topography. This phase component was estimated by using interferograms of the pairs of sessions, taken in the period of surface stability. Fig. 6 shows an example of subtraction the topographic phase on interferogram of a pair of sessions 20190208-20190220 from interferogram of a pair 20190115- 20190127. According to the corrected interferogram, (Fig. 6c), there are no any manifestation of small-scale dynamics of the landslide surface during period of blasting operations in the end of January. Similar results were obtained for interferograms of pairs 20190122-20190203, 20190127-20190208.

For estimation an amount of soil deposited by a landslide, let's take interferogram of the pair number 4 from the Table 1 covering period 3-15 January 2019, before the start of active blasting operations. Since we used for differential interferometric processing SRTM digital elevation model made long before the landslide event, the topography alteration after the landfall is clearly seen on the interferogram (Fig. 3c). The location of the landslide circus is noticeable here in a form of bright oval spot in the bottom half of the figure. Integration the phase difference within the landslide feature allowed us to estimate the amount of rock mass that was carried out. According to our estimates, made with account of $119 \mathrm{~m}$ height ambiguity for the interferometric pair used and the interferogram pixel square of 187 $\mathrm{m}^{2}$, the volume of the soil brought away by the landslide is 18.5 million $\mathrm{m}^{3}$. Maximal vertical displacement of the scattering surface because the landslide compared with SRTM model reached $140-150 \mathrm{~m}$, the landslide square in the vertical projection is 22.8 hectares.

\section{CONCLUSION}

Radar interferometry technique is an effective tool for measuring the parameters of the land surface and detection the small-scale dynamics of the underlying covers. Scale of the landslide event and the volume of rock soils brought away from the landslide slope on the Bureya River bank were estimated. It was found that the volume of soil removed because of landfall in December 2018 is at least 18.5 million $\mathrm{m}^{3}$, the landslide square in the vertical projection is 22.8 hectares. Maximal vertical displacement of the scattering surface relative to the SRTM digital elevation model after a landfall reached $150 \mathrm{~m}$. Despite the generally low quality of classical interferometric measurements by means of radars operating at such a relatively short wavelength band as on Sentinel-1, especially observing the areas with developed vegetation covers, it become possible to apply classical radar interferometry technique due to the availability of images taken during cold winter season, which provided a high temporal coherence of radar signals backscatter.

\section{REFERENCES}

1. DC FGBU NIC "PLANET". Space monitoring of the situation on the Bureya River 2018-12-24. https:/ /www.dvrcpod.ru/ News.php?id_new $=2821$.

2. Kramareva LS, Lupyan EA, Amelchenko YuA, Burtsev MA, Krasheninnikova YuS, Sukhanova VV, Shamilova YuA. Nablyudenie zony obrusheniya sopki $v$ rayone reki Bureya 11 dekabrya 2018 goda [Observation of the hill collapse zone near the Bureya River on December 11, 2018]. Sovremennye problemy distantsionnogo zondirovaniya Zemli iz kosmosa, 2018, 15(7):266-271 (in Russ.).

3. Colesanti C, Wasowski J. Investigating landslides with space-borne Synthetic Aperture Radar (SAR) interferometry. Engineering Geology, 2006, 88:173-199.

4. Fruneau B, Achache J, Delacourt C. Observation and Modeling of the SaintEtienne-de-Tinée Landslide Using SAR Interferometry. Tectonophysics, 1996, 265:181190.

5. Rott H, Scheuchl B, Siegel A, Grasemann 
B. SAR interferometry: a case study above the alts, Austria. Geophys. Res. Lett., 1999, 26:1629-1632.

6. Rott H, Mayer C, Siegel A. Alpine areas. Proc. International Conference on Synthetic Aperture Radar (EUSAR 2000), Munich, Germany, 2000.

7. Kimura H, Yamaguchi Y. Detection of landslide areas using satellite radar interferometry. Photogrammetric Engineering and Remote Sensing, 2000, 66:337-344.

8. Ferretti A, Prati C, Rocca F. Permanent scatterers in SAR interferometry. Proc. Geoscience and Remote Sensing Symposium (IGARSS 1999), Hamburg (Germany), 28 June-2 July 1999, vol. 3, pp. 1528-1530.

9. Ferretti A, Prati C, Rocca F. Nonlinear Subscription Rate for Permanent Scatterers in Differential SAR Interferometry. IEEE Trans. Geosci. Remote Sens., 2000, 38(5):22022212.

10. Colesanti C, Ferretti A, Prati C, Rocca F. Monitoring landslides and tectonic motion with the Permanent Scatterers technique. Engineering Geology, 2003, 68:3-14.

11. Colesanti C, Ferretti A, Novali F, Prati C, Rocca F. SAR monitoring of progressive and seasonal. ground deformation using the permanent scatterers technique. IEEE Trans. Geosci. Remote Sens., 2003, 41:1685-1701.

12. Ferretti A, Fumagalli A, Novali F, Prati C, Rocca F, Rucci A. A new algorithm for processing interferometric data-stacks: SqueeSAR. IEEE Trans. Geosci. Remote Sens., 2011, 49(9):3460-3470.

13. Hooper A, Zebker H, Segall P, Kampes B. Using InSAR persistent scatterers. Geophys. Res. Lett., 2004, 31(23).

14. Berardino P, Fornaro G, Lanari R, and Sansosti E. SAR interferograms. IEEE Trans. Geosci. Remote Sens., 2002, 40(11):2375-2383.

15. Rosen PA, Hensley S, Joughin IR, Li FK, Madsen SN, Rodriguez E, and Goldstein RM. Synthetic aperture radar interferometry. Proc. IEEE, 2000, 88 (3):333-392.
16. Zakharov AI, Yakovlev OI, Smirnov VM. Satellite monitoring of the Earth: Radar surface sensing. Moscow, URSS Publ., 2015, 248 p. 